\title{
Factor de impacto. Actas Urológicas Españolas
}

\author{
Actas Urol Esp 2005; 29 (9): 913-917
}

Estimado Sr. Director:

En el último número de Actas, que he recibido, abril de 2005 (Actas Urol Esp 2005; 29 (4): 345348), he leído con sorpresa un artículo del Dr. J.A. Lancina, titulado "En Defensa de la Expansión Internacional de Actas Urológicas Españolas”.

Más que un artículo científico, me ha parecido una antigua soflama patriótica, no se si buscando el beneficio de la Revista o el suyo propio, pero lo que está claro es que miente, ocultando datos.

Le incluyo una fotocopia de la VERDADERA, tabla del IHCD, respecto al factor de impacto, que por otra parte puede obtener cualquier lector en la dirección de Internet ime.uv.es/imecitas/ impcto.shtml y ime.uv.es/info/idex.htm.
Como se puede apreciar Archivos, está por encima de Actas, con un factor de impacto de 0,248, frente a Actas con un 0,186.

Espero que rectifique públicamente su falsa Tabla 2, de donde ha retirado a todas las Revistas, no puramente urológicas, lo cual veo lógico, pero también a Archivos Españoles de Urología.

Creo que sus lectores, debían enterarse de esta manipulación.

Esperando la publicación de esta carta, se despide atentamente,

Fdo.: Dr. E. Pérez-Castro Ellendt

Tabla del IHCD / Hoja 2

\begin{tabular}{|c|c|c|c|c|}
\hline \multirow[b]{2}{*}{ ISSN } & \multirow[b]{2}{*}{ Título Abreviado } & \multicolumn{2}{|c|}{ Total de 2000-2002 } & \multirow{2}{*}{$\begin{array}{c}\text { Factor de } \\
\text { Impacto }\end{array}$} \\
\hline & & Citas & Artículos & \\
\hline $1420-4096$ & Kidney \& Blood Pressure Research & 61 & 103 & 0,967 \\
\hline 0036-5599 & Scandinavian Jounal of Urology and Nephrology & 185 & 310 & 0,86 \\
\hline $1008-682 X$ & Asian Journal of Andrology & 89 & 161 & 0,836 \\
\hline $1078-1439$ & Urologic Oncology & 65 & 112 & 0,818 \\
\hline $0211-6995$ & Nefrología & 49 & 323 & 0,607 \\
\hline $0302-5144$ & Contributions to Hephrology & 95 & 209 & 0,549 \\
\hline $0042-1138$ & Urología Internationalis & 123 & 377 & 0,5 \\
\hline $0340-2592$ & Urologe-Ausgabe A & 70 & 200 & 0,496 \\
\hline $0090-2934$ & Dialysis \& Transplantation & 62 & 160 & 0,49 \\
\hline $0919-8172$ & International Journal of Urology & 131 & 404 & 0,488 \\
\hline $0886-022 \mathrm{X}$ & Renal Failure & 80 & 261 & 0,479 \\
\hline $1365-7852$ & Prostate Cancer and Prostatic Diseases & 42 & 127 & 0,458 \\
\hline $1166-7087$ & Progres en Urologie & 141 & 504 & 0,436 \\
\hline $0004-0614$ & Archivos Españoles de Urología & 77 & 425 & 0,248 \\
\hline $1320-5358$ & Nephrology & 22 & 174 & 0,243 \\
\hline $0250-4960$ & Nephrologie & 26 & 152 & 0,2 \\
\hline $0210-4806$ & Actas Urológicas Españolas & 56 & 412 & 0,186 \\
\hline $0003-4401$ & Annales d Urologie & 26 & 209 & 0,157 \\
\hline $0001-7868$ & Aktuelle Urologie & 19 & 245 & 0,089 \\
\hline $1464-4069$ & Bju International & 16 & 0 & 0 \\
\hline 0007-1331 & British Journal of Urology & 2 & 0 & 0 \\
\hline
\end{tabular}


Tabla 2

Factor de impacto (FI) de revistas de Urología

\begin{tabular}{|c|c|c|c|c|}
\hline Revista & $\mathbf{F} \mathbf{I}^{*}$ & Sociedad editora & País & Idioma \\
\hline Prostate & 3,167 & - & EE. UU. & Inglés \\
\hline Journal of Urology & 3,029 & American Urological Association & EE. UU. & Inglés \\
\hline Neurourology and Urodynamics & 2,560 & International Continence Society & EE. UU. & Inglés \\
\hline International Journal of Impotence Research & 2,544 & International Society for Sexual and impotence Research & Reino Unido & Inglés \\
\hline Urology & 2,506 & Societé Internationale d’Urologie & EE. UU. & Inglés \\
\hline The Urologic Clinics of North America & 2,340 & - & EE. UU. & Inglés \\
\hline European Urology & 1,889 & European Association of Urolofy & Paises Bajos & Inglés \\
\hline World Journal of Urology & 1,679 & - & EE. UU. & Inglés \\
\hline British Journal of Urology International & 1,640 & $\begin{array}{l}\text { British Association of Urologinecology Surgeons y Urological } \\
\text { Society of Australasia }\end{array}$ & Reino Unido & Inglés \\
\hline \multicolumn{5}{|l|}{ International Uroginecology Journal and Pelvis } \\
\hline Floor Dysfunction & 1,429 & $\begin{array}{l}\text { International Association of Uroginecology y Sociedad } \\
\text { Mexicana de Uroginecologia }\end{array}$ & Reino Unido & Inglés \\
\hline Molecular Urology & 1,310 & - & EE. UU. & Inglés \\
\hline Urological Research & 1,110 & - & EE. UU. & Inglés \\
\hline Journal of Endourology & 1,077 & Endourological Society y otras sociedades de Endourologia y ESWL & EE. UU. & Inglés \\
\hline Scandinavian Journal of Urology and Nephrology & 0,860 & $\begin{array}{l}\text { Scandinavian Association of Urology y otras sociedades } \\
\text { urológicas noreuropeas }\end{array}$ & Noruega & Inglés \\
\hline Asian Journal of Andrology & 0,836 & Asian Society of Andrology & China & Inglés \\
\hline $\begin{array}{l}\text { Urologic Oncology-Seminars and Original } \\
\text { Investigations }\end{array}$ & 0,818 & Society of Urologic Oncology & EE. UU. & Inglés \\
\hline Urologia Internationalis & 0,500 & - & Suiza & Inglés \\
\hline Urologe A & 0,496 & Deutschen Geselschaft fur Urologie & Alemania & Alemán \\
\hline Prostate Cancer and Prostatic Disease & 0,458 & - & Reino Unido & Inglés \\
\hline International Journal of Urology & 0,448 & Japanese Urological Association & Japón & Inglés \\
\hline Progres en Urologie & 0,436 & $\begin{array}{l}\text { Association Francaise d'Urologie, Association des Urologues } \\
\text { du Quebec y societé Belge d'Urologie }\end{array}$ & Francia & Francés \\
\hline Actas Urológicas Españolas & 0,186 & Asociación Española de Urología & España & Español \\
\hline Annales d'Urologie & 0,157 & - & Francia & $\begin{array}{l}\text { Francés/ } \\
\text { Inglés }\end{array}$ \\
\hline Aktuelle Urologie & 0,089 & - & Alemania & $\begin{array}{l}\text { Alemán / } \\
\text { Inglés }\end{array}$ \\
\hline
\end{tabular}

*FI adjudicado por el Institute for Scientific Information (año 2002), con adaptación para Actas Urológicas Españolas por el IHBD.

\section{RESPUESTA A LA CARTA DEL DR. PÉREZ-CASTRO ELLENDT}

\section{Estimado Sr. Director:}

En relación con el escrito remitido por el Dr. E. Pérez-Castro Ellendt, que hace referencia al artículo escrito por mí "En defensa de la expansión internacional de Actas Urológicas Españolas” publicado en la revista el pasado mes de abril, me veo en la obligación de hacer algunas aclaraciones.
En primer lugar, quiero manifestarle que me ratifico punto por punto en todos los contenidos del artículo, tanto en los objetivos propuestos como en la metodología seguida en la exposición de los hechos y en todas las conclusiones que se derivan.

Como queda claramente expresado en el texto del artículo, en la tabla 2 se pretende incluir a las "revistas de contenido urológico, general o específico, incluidas en el SCr' de las que desafortunadamente ninguna de ellas están "editadas en lengua española ni provenientes de paises his- 
panoparlantes", figurando el factor de impacto (FI) para cada revista "adjudicado para el año 2002" según se refiere en el pie de la tabla. En consecuencia, esta tabla pretende reflejar única y exclusivamente las revistas de contenido urológico (se excluyen revistas de Nefrología y otras especialidades) y que además están incluidas en el Sciencie Citation Index (SCI) cuyo FI publica el Journal Citation Report (JCR). Esta base de datos puede ser consultada en Internet (http://jcr9.isiknowledge.com), aunque de acceso restringido para abonados. Por tanto, en ningún momento se pretendía que en la tabla 2 quedasen reflejados los datos procedentes del Instituto de Historia de la Ciencia y Documentación (IHCD) de la Universidad de Valencia.

Por otra parte, según queda explicado en el artículo, la inclusión de Actas Urológicas Españolas dentro de esta tabla (en letra cursiva) solamente está justificada para "hacer una estimación del impacto actual" de Actas respecto a las revistas urológicas incluidas en el SCI, para lo cual "puede servir de referencia el FI que, para las revistas españolas, calcula el IHCD". Gueda claro, por tanto, que de todos los datos aportados por el IHCD, solamente se extrapola el FI adjudicado para Actas para estimar el impacto de la revista.

La ausencia en la tabla de Archivos Españoles de Urología y de otras revistas urológicas españolas obedece a dos motivos principales. En primer lugar, como ya ha sido referido, ninguna revista española de Urología está incluida en el SCI y, por tanto, no recibe el FI directamente del JCR. En segundo lugar, no se hizo para Archivos la extrapolación del FI que aporta el IHCD porque, como queda suficientemente aclarado, el artículo pretende analizar única y exclusivamente la situación de Actas Urológicas Españolas.

En consecuencia, de acuerdo con los objetivos que se persiguen con la publicación del artículo, la veracidad de los datos que se aportan y después de hacer todas estas aclaraciones, que creo no eran necesarias pues quedan suficientemente aclaradas con la simple lectura del artículo, rechazo tajantemente las atribuciones que se me imputan de "mentir", "ocultar datos" o "manipular”.

No tengo por costumbre hacer referencia a aspectos que se aparten de los propios contenidos que se expresan en los escritos, pero de forma excepcional, en relación con las especulaciones que se dejan caer sobre los posibles "beneficios" que se quieren buscar con la publicación de este artículo, diré que el único interés que me motivó para su escritura fue aportar una modesta contribución para que pueda mejorar la calidad científica y difusión de Actas Urológicas Españolas, la revista que es Órgano Oficial de Difusión de la Asociación Española de Urología sin ningún ánimo de lucro, y todo ello en mi condición de socio de la AEU y miembro del Comité de Revisores de la revista.

Para finalizar, Sr. Director, quisiera expresarle que, a poco que se profundice en la lectura del artículo, se verá que está lleno de mensajes de unidad, trabajo cooperativo y en la búsqueda de puentes de entendimiento entre todos los que estamos interesados en mejorar la calidad e impacto de las revistas publicadas en países hispanos, objetivo que además de necesario también es legítimo.

Dr. J.A. Lancina Martín

Servicio de Urología

Hospital Juan Canalejo

Xubias de Arriba, 84

15006 A Coruña

\section{CONSIDERACIONES AL RESPECTO DEL DIRECTOR DE ACTAS UROLÓGICAS ESPAÑOLAS}

En este número de Actas Urológicas Españolas se publica la carta que el Dr. Enrique Peréz-Castro Ellendt, director de la revista Archivos Españoles de Urología, mandó a esta dirección con relación al artículo escrito por el Dr. Juan Alberto Lancina Martín titulado "En defensa de la expansión internacional de Actas Urológicas Españolas” y publicado en el número de Abril de este año (Actas Urol Esp 2005, 29 (4):345-348). El Dr. Pérez-Castro Ellendt se queja de que, en el citado artículo, se falta a la verdad, se ocultan datos y se manipula la información sobre el factor de impacto que la revista Archivos Españoles de Urología tiene respecto a Actas Urológicas Españolas. Aporta una dirección de internet (ime.uv.es/imecitas/impcto/shtml e ime.uv.es/info/idex.html) donde se puede encon- 
trar la verdadera información y comprobar que el factor de impacto de Archivos Españoles de Urología es superior al de Actas Urológicas Españolas. Por todo ello, solicita una rectificación pública y la publicación de su carta.

Es evidente que la carta que el Dr. Lancina ha escrito como contestación a la del Dr. PérezCastro, no necesita ninguna aclaración, apoyo, defensa ni puntualización. Sin embargo, si creo es mi obligación, como director actual de Actas Urológicas Españolas, dar mi opinión al respecto y realizar algunos comentarios.

El Journal Citation Index es el sistema de referencia para establecer la repercusión científica y el factor de impacto que tiene una revista a nivel internacional. Es evidente que la lengua inglesa domina el mundo de las publicaciones científicas. La gran mayoría de revistas científicas con elevado factor de impacto están escritas en lengua inglesa. Si bien, existen publicaciones de elevado prestigio en lengua no inglesa, lo cierto es que, lo que no está publicado en inglés apenas tiene repercusión. Por este motivo las revistas científicas españolas tienen difícil, no sólo conseguir un elevado factor de impacto, sino ser evaluadas y aparecer en el Journal Citation Report. Los datos a los que hace referencia el Dr. Pérez-
Castro Ellendt proceden de El Instituto de Historia de la Ciencia y Documentación López Piñero de la Universidad de Valencia. Este organismo lleva a cabo un proyecto financiado por la Subdirección General de Estudios y Análisis del Ministerio de Educación, Cultura y Deporte, para el estudio del "Factor de impacto potencial de las revistas médicas españolas". Su objetivo principal es determinar el factor de impacto nacional e internacional de las revistas biomédicas españolas. Este proyecto se lleva realizando desde 1999. El cálculo del factor de impacto es bianual. Por lo tanto, únicamente hay datos de los bienios 19992001, 2000-2002, 2001-2003 para el factor de impacto nacional. Para el factor de impacto internacional hay datos de los años 2001 y 2002, de forma aislada, y global para el bienio 2001-2003. Como demuestra la tabla adjunta, el valor del factor de impacto de una revista científica es dinámico y cambiante. Por lo que, para conocer realmente el valor científico de una revista, tiene más valor la evolución y tendencia a lo largo de los años que las estimaciones puntuales.

Los valores de factor de impacto que el Dr. Pérez-Castro Ellendt atribuye a ambas revistas son rigurosamente ciertos pero parciales. En primer lugar, porque corresponden a sólo uno de los

Tabla

Comparación de factor de impacto nacional e internacional por años considerados por El Instituto de Historia de la Ciencia y Documentación López Piñero para Actas Urológicas Españolas y Archivos Españoles de Urología

\begin{tabular}{|c|c|c|c|c|c|c|}
\hline Ámbito & Revista & Años & Citas & Artículos & Factor de impacto & $\begin{array}{l}\text { Índice de } \\
\text { Inmediatez }\end{array}$ \\
\hline \multirow[t]{6}{*}{ Nacional } & *AUE & 1999-2001 & 68 & 436 & 0,220 & 0,008 \\
\hline & $* \mathrm{AEU}$ & 1999-2001 & 48 & 457 & 0,152 & 0,007 \\
\hline & AUE & 2000-2002 & 44 & 412 & 0,144 & 0,017 \\
\hline & AEU & 2000-2002 & 53 & 425 & 0,169 & 0,016 \\
\hline & AUE & 2001-2003 & 44 & 386 & 0,173 & 0 \\
\hline & AUE & 2001-2003 & 29 & 410 & 0,103 & 0,007 \\
\hline \multicolumn{7}{|c|}{ Internacional } \\
\hline & AUE & 2001 & 82 & 436 & 0,266 & 0,008 \\
\hline & AEU & 2001 & 81 & 457 & 0,255 & 0,014 \\
\hline & AUE & 2002 & 56 & 412 & 0,186 & 0,017 \\
\hline & AEU & 2002 & 77 & 425 & 0,248 & 0,016 \\
\hline & AUE & 2001-2003 & 56 & 386 & 0,221 & 0 \\
\hline & AUE & 2001-2003 & 52 & 410 & 0,188 & 0,007 \\
\hline
\end{tabular}

*AUE: Actas Urológicas Españolas

*AEU: Archivos Españoles de Urología 
bienios considerados (2000-2002) en la estimación y, en segundo, porque son datos únicamente relacionados con el factor de impacto internacional. Como se puede apreciar en la tabla adjunta, en todos los demás bienios considerados, independientemente del ámbito nacional o internacional, el valor del factor de impacto es superior en Actas Urológicas Españolas que en Archivos Españoles de Urología.

La situación de las revistas nacionales europeas pasa por un momento delicado. La hegemonía de la lengua inglesa en la literatura científica urológica es aplastante. Hace unos años el Journal of Urology era la revista urológica más consultada. Recientemente el European Urology ha ido ganando en factor de impacto y ha conseguido mitigar, de algún modo, el predominio de la revista americana. Esta mejoría ha llevado como consecuencia un debilitamiento de las revistas nacionales. La revista de urología francesa ha desaparecido y la italiana también, nombrando al European Urology su órgano oficial de difusión. En la actualidad, de los grandes países europeos, se mantienen la revista alemana y la nuestra. Por lo que, el futuro y viabilidad de las revistas científicas nacionales están seriamente comprometidas.

En la actualidad, Archivos Españoles de Urología y Actas Urológicas Españolas son las dos revistas más importantes de urología de nuestro país.
Ni dispongo de la información ni me corresponde analizar las circunstancias históricas por las que la literatura científica urológica española ha evolucionado a través de estas dos revistas. Lo cierto es que, posiblemente, en toda Europa no exista un país con dos publicaciones de tan buen nivel científico y, lo que es más importante, en una lengua con verdadera proyección internacional. Como así lo demuestra la expansión que ha sufrido en los últimos años el idioma español. Por lo que, más que entrar en una discusión de números y datos, me gustaría establecer una competencia sana y en términos científicos que sea estimulante y enriquecedora. Siempre, desde el mayor de los respetos y admiración por todo lo que representa Archivos Españoles de Urología.

Por último quiero expresar mi gratitud al Dr. Juan Alberto Lancina por su compromiso sincero, bien intencionado y totalmente desinteresado con nuestra revista. Desde el Comité Editorial, ya que supone un ejemplo por su implicación constante en el desarrollo y mejora de la misma.

Dr. J.L. Ruiz Cerdá

Director de Actas Urológicas Españolas 